\title{
Combination of D-dimer level and neutrophil to lymphocyte ratio predicts long-term clinical outcomes in acute coronary syndrome after percutaneous coronary intervention
}

\author{
Ling-Feng Gu, Jie Gu, Si-Bo Wang, Hao Wang, Ya-Xin Wang, Yuan Xue, Tian-Wen Wei, \\ Jia-Teng Sun, Xiao-Qing Lian, Jia-Bao Liu, En-Zhi Jia, Lian-Sheng Wang
}

Department of Cardiology, the First Affiliated Hospital of Nanjing Medical University, Nanjing, China

\begin{abstract}
Background: High D-dimer (DD) is associated with short-term adverse outcomes in patients with acute coronary syndrome (ACS). In ACS patients who underwent percutaneous coronary intervention (PCI), however, the value of DD (or combined with neutrophil to lymphocyte ratio [NLR]) to predict long-term major adverse cardiovascular events (MACEs) has not been fully evaluated.

Methods: Patients diagnosed with ACS and receiving PCI were included. The primary outcome was MACEs. Cox proportional hazards regression and logistic regression were used to illustrate the relationship between clinical risk factors, biomarkers and MACEs. Survival models were developed based on significant factors and evaluated by the Concordance-index (C-index).

Results: The final study cohort was comprised of 650 patients (median age, 64 years; 474 males), including 98 (15\%) with MACEs during a median follow-up period of 40 months. According to the cut-off value of $D D$ and NLR, the patients were separated into four groups: high DD or nonhigh DD with high or nonhigh NLR. After adjusting for confounding variables, DD (adjusted hazard ratio [aHR]: 2.39, 95\% confidence interval [CI]: 1.52-3.76) and NLR (aHR: 2.71, 95\% CI: 1.78-4.11) were independently associated with long-term MACEs. Moreover, patients with both high DD and NLR had a significantly higher risk in MACEs when considering patients with nonhigh DD and NLR as reference (aHR: 6.19, 95\% CI: 3.30-11.61). The area under curve increased and reached 0.70 in differentiating long-term MACEs when DD and NLR were combined, and survival models incorporating the two exhibited a stronger predictive power (C-index: 0.75).

Conclusions: D-dimer (or combined with NLR) can be used to predict long-term MACEs in ACS patients undergoing PCI. (Cardiol J)
\end{abstract}

Key words: acute coronary syndrome, D-dimer, neutrophil to lymphocyte ratio, long-term outcome, percutaneous coronary intervention

\section{Introduction}

Coronary heart disease (CHD) is one of the leading causes of death worldwide [1]. Acute coro- nary syndrome (ACS) is an acute manifestation of CHD, including ST-segment elevation myocardial infarction (STEMI), non-ST-segment elevation myocardial infarction (NSTEMI) and unstable

Address for correspondence: Lian-Sheng Wang, MD, PhD and En-Zhi Jia, MD, PhD, Department of Cardiology, the First Affiliated Hospital of Nanjing Medical University, Nanjing, 300 Guangzhou Road, Nanjing, 210029, Jiangsu Province, China, tel/fax: 0086-25-83724440, e-mail: drlswang@njmu.edu.cn; e-mail: enzhijia@njmu.edu.cn

Received: 28.02.2021 Accepted: 6.08.2021 Early publication date: 17.08.2021

This article is available in open access under Creative Common Attribution-Non-Commercial-No Derivatives 4.0 International (CC BY-NC-ND 4.0) license, allowing to download articles and share them with others as long as they credit the authors and the publisher, but without permission to change them in any way or use them commercially. 
angina, which has a higher risk of morbidity, rehospitalization, and mortality. Thus, adequate risk assessments are essential for patients with ACS.

D-dimer (DD) is a fibrin degradation product during the process of fibrinolysis, and is often used as a marker for hypercoagulation and thrombus formation. Many diseases present with elevated $\mathrm{DD}$, such as venous thromboembolism, acute aortic dissection and peripheral artery disease. Recently, it has been discovered that the above-mentioned diseases may not be the only scenarios where DD can be used. Studies revealed that baseline DD can be utilized as a predictive biomarker for cardiovascular outcomes in patients with stable CHD and ACS $[2,3]$. However, its clinical efficacy in long-term cardiovascular outcomes has not yet been fully assessed in patients with ACS receiving percutaneous coronary intervention (PCI).

Atherosclerosis is an inflammatory disease and it plays a dominant role in ACS $[4,5]$. Lately, inflammatory biomarkers such as neutrophil to lymphocyte ratio (NLR) have emerged to be easily accessible, cost-effective indicators in various cardiovascular disorders including stable CHD, heart failure and ACS [6-10]. Previous studies have shown that NLR was an independent predictor of major adverse cardiovascular events (MACEs) in patients with ACS $[7,8]$.

This study tried to evaluate the long-term prognostic value of DD alone and in combination with NLR for MACEs in patients with ACS who underwent PCI.

\section{Methods}

\section{Study design and population}

This was a retrospective longitudinal cohort study which included 792 consecutive patients who were diagnosed as ACS at the Department of Cardiology, First Affiliated Hospital of Nanjing Medical University between February 2016 and December 2016.

Acute coronary syndrome was subdivided into STEMI, NSTEMI and unstable angina, and the diagnostic criteria were as follows. STEMI was considered in the presence of (1) at least two contiguous leads with ST-segment elevation $\geq 2 \mathrm{~mm}$ in chest leads and/or ST-segment elevation of $\geq 1 \mathrm{~mm}$ in the other leads or new left bundle branch block (LBBB) and (2) a detection of a dynamic change of cardiac troponin $T$ with at least one value above the $99^{\text {th }}$ percentile upper reference limit $[11,12]$. If electrocardiograph revealed diagnostic ischemia patterns without persistent ST-elevation as well as cardiac markers were elevated, patients were considered to have NSTEMI. Those who had typical chest pain and cardiac markers within the normal range were deemed to have unstable angina [13]. All three main coronary arteries (left anterior descending coronary artery and/or collateral, left circumflex coronary artery and/or collateral, right coronary artery and/or collateral) had at least one stenosis (diameter stenosis determined by angiography $\geq 50 \%$ ), with or without the left main artery involvement was considered to be a three-vessel or left main disease. Other definitions in the present study including hypertension, diabetes, dyslipidemia, smoking and drinking were based on currently accepted criteria.

The inclusion criteria were: (1) adult patients (age older than 18 years), (2) clinically diagnosed as ACS and underwent PCI. The exclusion criteria were as follows: (1) patients were on anticoagulant or steroid therapy at admission (10 cases), (2) patients had active inflammation at admission (defined as $\mathrm{T} \geq 38.5^{\circ} \mathrm{C}$ and at least one typical symptom, e.g. cough, sneezing or vomiting) or experienced vascular thromboembolism within 6 months (24 cases), (3) patients with comorbidities, including hematological disorders, uncontrolled chronic inflammation disease, malignant tumors, severe hepatic disease or renal disease (alanine aminotransferase $>400 \mathrm{U} / \mathrm{L}$ or estimated glomerular filtration rate $[\mathrm{eGFR}]<30 \mathrm{~mL} / \mathrm{min} / 1.73 \mathrm{~m}^{2}$ ) (50 cases), (4) blood cell count or DD not collected before PCI (5 cases). Due to follow-up data for $53(7.5 \%)$ patients were not available, a total of 650 patients were included in the final cohort for analysis.

\section{Medical variables collection}

Patients' gender, age, body mass index (BMI), admission and discharge date, smoking, drinking history, hypertension, diabetes, and laboratory measurements, procedural details and medications at discharge were collected. Information of these variables was obtained from the hospital information system or by contacting patients or their family members.

\section{Laboratory tests}

Venous blood samples were obtained within $24 \mathrm{~h}$ before the initial treatment for blood analysis. Whole blood cell counts including leucocytes, neutrophils, lymphocytes, hemoglobin and platelets were measured using the XE-5000 fully-autonomic hematology analyzer (Sysmex, Kobe, Japan). NLR was calculated by dividing the neutrophil count 
by the lymphocyte count. Biochemical indicators including total cholesterol, triglycerides, low-density lipoprotein cholesterol (LDL-C), high-density lipoprotein cholesterol (HDL-C), liver and renal function, cardiac troponin $\mathrm{T}$ were determined using an automatic biochemistry analyzer (Bayer ADVIA 1650, Siemens, Wilmington, DE, USA). Plasma DD was carried out by immunoturbidimetric assay using a coagulation analyzer (Diamed CDX, Diamond Diagnostics, Holliston, USA).

\section{Clinical follow-up and outcomes}

Follow-up was performed by trained coordinators through telephone interviews or review of medical records. The primary outcomes of this study were the occurrence of MACEs, including all-cause mortality, cardiac death, myocardial infarction (MI), target vessel revascularization (TVR) and stroke during follow-up. Cardiac death was defined as any death caused by cardiac problems. MI was defined based on the fourth universal definition of MI [11]. Stroke was defined as suddenly developed sensory and motor dysfunction resulting from brain abnormalities, or imaging studies revealed evidences of brain infarction or hemorrhage related to the symptoms [14]. TVR was defined as any repeat intervention of upstream and downstream branches of the target vessel and itself as recommended by the Academic Research Consortium [15].

\section{Ethical approval}

This study protocol was approved by the Ethics Committee of First Affiliated Hospital of Nanjing Medical University. The need for informed consent from patients was waived because of the retrospective study design.

\section{Statistical analysis}

Continuous variables were expressed as median and interquartile range (IQR). Categorical variables were expressed as numbers (percentages). Continuous variables were compared using the Mann-Whitney U test or Kruskal-Wallis $\mathrm{H}$ test as appropriate and categorical variables were compared using the chi-square $\left(\chi^{2}\right)$ test or the Fisher exact test.

Receiver operating characteristic (ROC) curves were plotted to calculate the area under curves (AUC), cut-off values, sensitivities, and specificities of DD and NLR. In accordance with the optimal cut-off values of DD $(0.31 \mu \mathrm{g} / \mathrm{mL})$ and NLR (3.31), patients were divided into four groups: nonhigh DD and nonhigh NLR (DD-/NLR-), non- high DD and high NLR (DD-/NLR+), high DD and nonhigh NLR (DD+/NLR-), and high DD and high NLR $(\mathrm{DD}+/ \mathrm{NLR}+)$. The Delong test (AUC comparison), net reclassification index (NRI), and integrated discrimination index (IDI) were evaluated for model discrimination.

To identify and evaluate the independent predictors of MACEs, univariate and multivariate Cox proportional hazards analyses were obtained. Variables including age, gender, BMI, comorbidities, known medical history, biomarkers, medications and procedural data were adjusted in this model. Logistic regression was also conducted to validate the independent predictive factors of MACEs. Different survival models were developed basing on identified variables predictive for MACEs. The Concordance index (C-index) was calculated to evaluate the discriminative ability of each model. The $\mathrm{p}$ value from the likelihood-ratio test was used for comparison of different models. The results of the risk analyses were provided as hazard ratios with $95 \%$ confidence interval (CI) and the results of logistic regression were shown as the odds ratio and $95 \%$ CI. P value $<0.05$ (two-tailed) was considered statistically significant. Statistical analysis was performed using IBM SPSS 24.0 software package (SPSS Inc., Chicago, Illinois, USA) and GraphPad Prism version 8.0.0 for Windows (GraphPad Software, San Diego, California USA).

\section{Results}

\section{Baseline clinical characteristics}

A total of 792 patients were screened, of whom 650 patients met the criteria for enrollment (Fig. 1). The clinical characteristics of the entire study cohort are summarized in Table 1 . The median age of the study cohort was 64 years, and $73 \%$ were male. Among all patients, $68 \%$ had hypertension, $22 \%$ had diabetes and 5\% had a history of PCI. Compared to the MACEs-free group, the patients in the MACEs group were older, had higher levels of DD, NLR, leucocytes, neutrophils, peak cardiac troponin $\mathrm{T}$, and a higher proportion of three-vessel or left main disease. They also presented with lower levels of lymphocytes, eGFR, and had poorer ejection fraction (EF).

Patients with higher levels of DD and NLR tended to have worse prognoses. ROC curves were then used to determine the cut-off values of DD and NLR, and patients were divided into four groups according to the levels of DD and NLR for further analysis. Table 2 shows that patients in the high DD groups were more likely to be elderly, had lower 


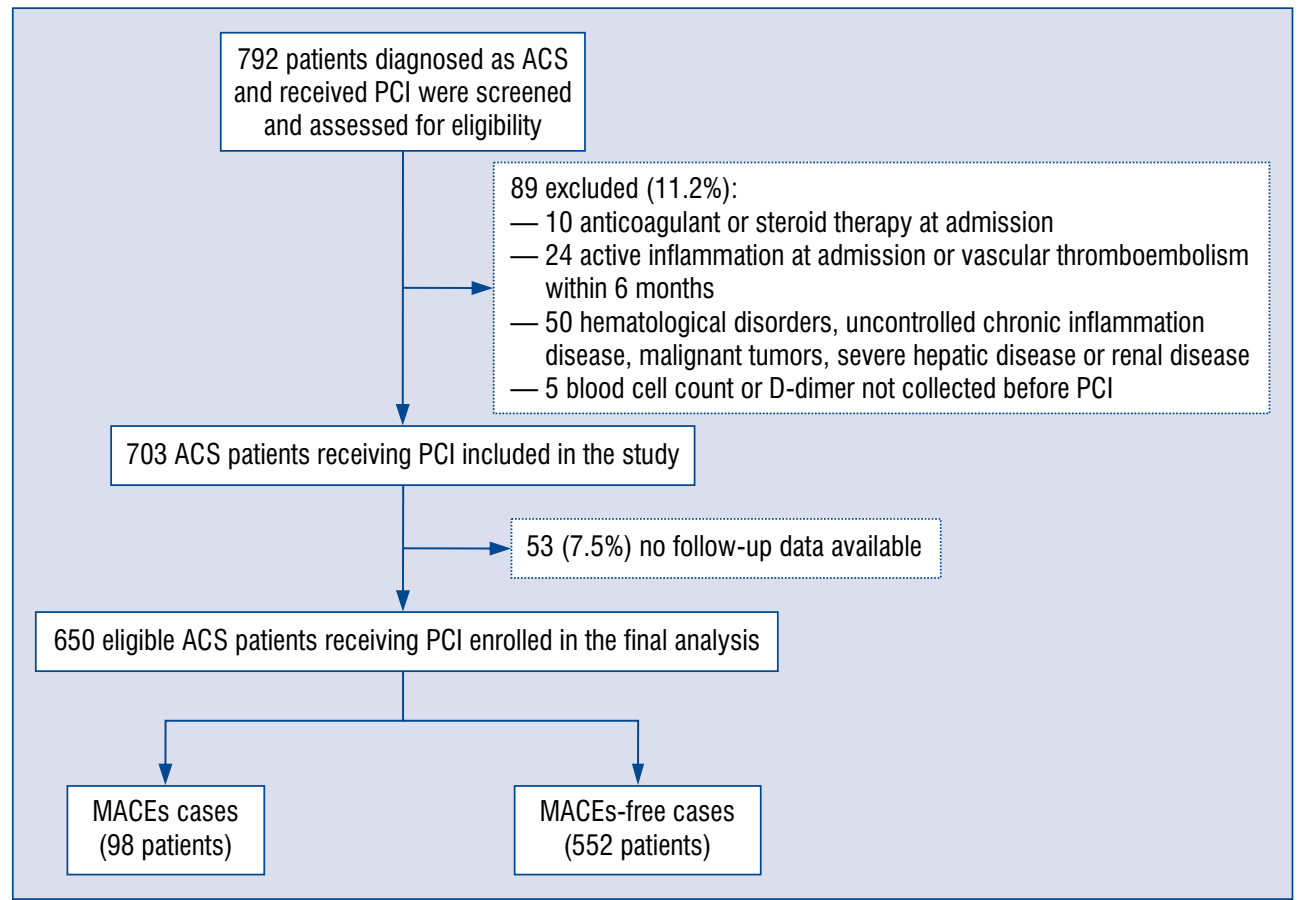

Figure 1. Flow chart of patient inclusion; ACS - acute coronary syndrome; $\mathrm{PCl}$ - percutaneous coronary intervention; MACEs - major adverse cardiovascular events.

Table 1. Comparison of baseline characteristics between patients with major adverse cardiovascular events (MACEs)-free and MACEs groups.

\begin{tabular}{|c|c|c|c|c|}
\hline Characteristics & $\begin{array}{c}\text { Total } \\
(n=650)\end{array}$ & $\begin{array}{l}\text { MACEs-free } \\
(n=552)\end{array}$ & $\begin{array}{l}\text { MACEs } \\
(n=98)\end{array}$ & $\mathbf{P}$ \\
\hline Age [years] & $64(56-71)$ & $63(55-70)$ & $67(61-75)$ & $<0.001$ \\
\hline Gender, Male & $474(72.9 \%)$ & $398(72.1 \%)$ & $76(77.6 \%)$ & 0.263 \\
\hline BMI $\left[\mathrm{kg} / \mathrm{m}^{2}\right]$ & $24.7(22.9-26.6)$ & $24.8(22.9-26.6)$ & $24.1(22.9-26.1)$ & 0.108 \\
\hline Hypertension & $440(67.7 \%)$ & $373(67.6 \%)$ & $67(68.4 \%)$ & 0.877 \\
\hline Diabetes mellitus & $161(21.8 \%)$ & $140(25.4 \%)$ & $21(21.4 \%)$ & 0.406 \\
\hline Dyslipidemia & $449(69.1 \%)$ & $80(70.2 \%)$ & $69(70.4 \%)$ & 0.757 \\
\hline Smoking & $236(36.3 \%)$ & $199(36.1 \%)$ & $37(37.8 \%)$ & 0.746 \\
\hline Drinking & $108(16.6 \%)$ & $94(17.0 \%)$ & $14(14.3 \%)$ & 0.501 \\
\hline Previous PCl & $32(4.9 \%)$ & $26(4.7 \%)$ & $6(6.1 \%)$ & 0.610 \\
\hline \multicolumn{5}{|l|}{ Laboratory parameters } \\
\hline Leucocyte $\left[10^{9} / \mathrm{L}\right]$ & 6.77 (5.63-8.93) & 6.67 (5.58-8.57) & $7.47(5.78-11.26)$ & 0.003 \\
\hline Neutrophil $\left[10^{9} / \mathrm{L}\right]$ & $4.47(3.36-6.19)$ & $4.32(3.32-5.80)$ & $5.39(3.71-8.76)$ & $<0.001$ \\
\hline Lymphocyte [10\%/L] & $1.70(1.30-2.13)$ & $1.74(1.34-2.15)$ & $1.49(1.07-1.98)$ & $<0.001$ \\
\hline NLR & $2.46(1.82-3.96)$ & $2.36(1.80-3.53)$ & $3.92(2.10-6.83)$ & $<0.001$ \\
\hline $\mathrm{D}$-dimer $[\mu \mathrm{g} / \mathrm{mL}]$ & $0.24(0.14-0.42)$ & $0.22(0.14-0.38)$ & $0.37(0.23-0.83)$ & $<0.001$ \\
\hline Total cholesterol [mmol/L] & $4.20(3.52-4.99)$ & $4.17(3.50-5.01)$ & 4.35 (3.60-4.95) & 0.547 \\
\hline Triglycerides [mmol/L] & $1.32(1.00-1.76)$ & $1.33(0.99-1.78)$ & $1.32(1.01-1.66)$ & 0.926 \\
\hline LDL-C [mmol/L] & $2.71(2.20-3.25)$ & $2.69(2.17-3.25)$ & $2.80(2.28-3.32)$ & 0.364 \\
\hline HDL-C [mmol/L] & $1.01(0.87-1.19)$ & $1.02(0.87-1.19)$ & $0.95(0.86-1.19)$ & 0.146 \\
\hline eGFR $\left[\mathrm{mL} / \mathrm{min} / 1.73 \mathrm{~m}^{2}\right]$ & $93.00(83.00-100.00)$ & $93.00(84.00-101.00)$ & 89.00 (76.75-99.00) & 0.012 \\
\hline Peak cTnT [ng/L] & $\begin{array}{c}20.05 \\
(7.86-1032.50)\end{array}$ & $\begin{array}{c}15.15 \\
(7.56-667.50)\end{array}$ & $\begin{array}{c}492.20 \\
(14.73-3004.75)\end{array}$ & $<0.001$ \\
\hline Ejection fraction [\%] & $63.00(60.10-65.40)$ & $63.30(60.90-65.60)$ & $61.95(51.75-64.00)$ & $<0.001$ \\
\hline
\end{tabular}


Table 1 (cont.). Comparison of baseline characteristics between patients with major adverse cardiovascular events (MACEs)-free and MACEs groups.

\begin{tabular}{lcccc}
\hline Characteristics & $\begin{array}{c}\text { Total } \\
(\mathbf{n}=650)\end{array}$ & $\begin{array}{c}\text { MACEs-free } \\
(\mathbf{n}=552)\end{array}$ & $\begin{array}{c}\text { MACEs } \\
(\mathbf{n}=98)\end{array}$ & P \\
\hline Medical treatment & & & & \\
Antiplatelet & $647(99.5 \%)$ & $550(99.6 \%)$ & $97(99.0 \%)$ & 0.388 \\
Antihypertensive & $542(83.4 \%)$ & $461(83.5 \%)$ & $81(82.7 \%)$ & 0.833 \\
Lipid-lowering medications & $642(98.8 \%)$ & $546(98.9 \%)$ & $96(98.0 \%)$ & 0.345 \\
Procedural data & & & & \\
Vessel treated: & $10(1.5 \%)$ & $6(1.1 \%)$ & $4(4.1 \%)$ & 0.049 \\
$\quad$ LM & $449(69.1 \%)$ & $386(69.9 \%)$ & $63(64.3 \%)$ & 0.265 \\
LAD & $210(32.3 \%)$ & $175(31.7 \%)$ & $35(35.7 \%)$ & 0.434 \\
LCX & $257(39.5 \%)$ & $215(38.9 \%)$ & $42(42.9 \%)$ & 0.466 \\
RCA & $296(45.5 \%)$ & $240(43.5 \%)$ & $56(57.1 \%)$ & 0.012 \\
Three-vessel or left main & $2.00(1.00-2.00)$ & $2.00(1.00-2.00)$ & $2.00(1.00-3.00)$ & 0.097 \\
Number of implanted stents & $40.50(24.75-60.00)$ & $39.00(24.00-59.00)$ & $48.00(25.00-66.00)$ & 0.052 \\
Total stent length [mm] & $5.25(3.00-7.25)$ & $5.25(3.00-7.00)$ & $5.75(3.00-7.75)$ & 0.399 \\
Total stent diameter [mm] & & & & \\
\hline
\end{tabular}

Data are median (interquartile range) or number (\%). BMI — body mass index; cTnT — cardiac troponin T; eGFR, — estimated glomerular filtration rate; HDL-C — high-density lipoprotein cholesterol; LAD — left anterior descending coronary artery; LCX - left circumflex coronary artery; LDL-C — low-density lipoprotein cholesterol; LM — left main coronary artery; MACEs — major adverse cardiovascular events; $\mathrm{NLR}$ - neutrophil to lymphocyte ratio; $\mathrm{PCl}$ - percutaneous coronary intervention; RCA — right coronary artery

Table 2. Characteristics in the four groups according to the cut-off values of D-dimer and NLR.

\begin{tabular}{|c|c|c|c|c|c|}
\hline Characteristics & $\begin{array}{c}\text { DD-/NLR- } \\
(n=300)\end{array}$ & $\begin{array}{c}\text { DD-/NLR+ } \\
(n=114)\end{array}$ & $\begin{array}{c}\text { DD + /NLR- } \\
(n=141)\end{array}$ & $\begin{array}{c}\text { DD +/NLR+ } \\
(\mathrm{n}=95)\end{array}$ & $\mathbf{P}$ \\
\hline Age [years] & $61(53-67)$ & $62(53-71)$ & $69(62-75)$ & $69(61-78)$ & $<0.001$ \\
\hline Male & $211(70.3 \%)$ & $101(88.6 \%)$ & $92(65.2 \%)$ & $70(73.7 \%)$ & $<0.001$ \\
\hline BMI $\left[\mathrm{kg} / \mathrm{m}^{2}\right]$ & $24.8(22.9-26.7)$ & $24.6(23.0-26.5)$ & $24.6(22.7-26.7)$ & $24.2(22.7-26.4)$ & 0.459 \\
\hline Hypertension & $197(65.7)$ & $82(71.9)$ & $100(70.9)$ & $61(64.2)$ & 0.441 \\
\hline Diabetes mellitus & $71(23.7 \%)$ & $18(15.8 \%)$ & $48(34 \%)$ & $24(25.3 \%)$ & 0.009 \\
\hline Dyslipidemia & $213(71.0 \%)$ & $80(70.2 \%)$ & $99(70.2 \%)$ & $57(60.0 \%)$ & 0.228 \\
\hline Smoking & $107(35.7 \%)$ & $51(44.7 \%)$ & $44(31.2 \%)$ & $34(35.8 \%)$ & 0.161 \\
\hline Drinking & $60(20.0 \%)$ & $18(15.8 \%)$ & $18(12.8 \%)$ & $12(12.6 \%)$ & 0.162 \\
\hline Previous $\mathrm{PCl}$ & $13(4.3 \%)$ & $4(3.5 \%)$ & $8(5.8 \%)$ & $7(7.4 \%)$ & 0.527 \\
\hline \multicolumn{6}{|l|}{ Laboratory parameters } \\
\hline Leukocyte $\left[10^{9} / \mathrm{L}\right]$ & $6.30(5.28-7.52)$ & $9.56(7.03-11.37)$ & $6.03(4.92-7.43)$ & $9.13(6.94-12.22)$ & $<0.001$ \\
\hline Neutrophil $\left[10^{9} / \mathrm{L}\right]$ & $3.88(2.97-4.72)$ & 7.49 (5.28-9.37) & $3.68(2.90-4.56)$ & 7.04 (5.45-10.39) & $<0.001$ \\
\hline Lymphocyte $\left[10^{9} / \mathrm{L}\right]$ & $1.90(1.59-2.31)$ & $1.30(0.99-1.69)$ & $1.85(1.49-2.23)$ & $1.21(1.01-1.47)$ & $<0.001$ \\
\hline NLR & $2.04(1.55-2.50)$ & $5.45(4.02-8.17)$ & $2.08(1.60-2.45)$ & $5.48(4.06-8.17)$ & $<0.001$ \\
\hline D-dimer $[\mu \mathrm{g} / \mathrm{mL}]$ & $0.17(0.11-0.23)$ & $0.16(0.11-0.23)$ & $0.51(0.37-0.81)$ & $0.62(0.45-1.24)$ & $<0.001$ \\
\hline Total cholesterol [mmol/L] & $4.10(3.45-4.96)$ & $4.34(3.55-5.13)$ & $4.14(3.40-4.88)$ & $4.41(3.69-5.12)$ & 0.085 \\
\hline Triglycerides (mmol/L] & $1.36(1.00-1.99)$ & $1.20(0.99-1.65)$ & $1.32(1.01-1.66)$ & $1.31(0.90-1.65)$ & 0.174 \\
\hline LDL-C [mmol/L] & $2.65(2.09-3.21)$ & $2.83(2.22-3.48)$ & $2.72(2.16-3.25)$ & $2.73(2.28-3.47)$ & 0.106 \\
\hline HDL-C [mmol/L] & $1.01(0.86-1.19)$ & $1.02(0.87-1.17)$ & $1.00(0.84-1.20)$ & $1.06(0.92-1.23)$ & 0.242 \\
\hline eGFR $\left[\mathrm{mL} / \mathrm{min} / 1.73 \mathrm{~m}^{2}\right]$ & $96.0(88.0-102.0)$ & $95.0(85.0-101.50)$ & $85.0(76.0-95.0)$ & $87.0(75.0-98.0)$ & $<0.001$ \\
\hline Peak cTnT [ng/L] & $10(5-43)$ & $1375(125-4495)$ & $15(8-157)$ & $1926(74-4982)$ & $<0.001$ \\
\hline Ejection fraction [\%] & $64.0(62.0-65.9)$ & $62.05(56.0-65.5)$ & $62.1(60.0-64.4)$ & $60.9(51.0-63.7)$ & $<0.001$ \\
\hline
\end{tabular}


Table 2 (cont.). Characteristics in the four groups according to the cut-off values of D-dimer and NLR.

\begin{tabular}{|c|c|c|c|c|c|}
\hline Characteristic & $\begin{array}{l}\text { DD-/NLR- } \\
(n=300)\end{array}$ & $\begin{array}{c}\text { DD-/NLR+ } \\
(\mathrm{n}=114)\end{array}$ & $\begin{array}{c}\text { DD + /NLR- } \\
(\mathrm{n}=141)\end{array}$ & $\begin{array}{c}\text { DD + /NLR+ } \\
(\mathrm{n}=95)\end{array}$ & $\mathbf{P}$ \\
\hline \multicolumn{6}{|l|}{ Medical treatment } \\
\hline Antiplatelet & $299(99.7 \%)$ & $113(99.1 \%)$ & $140(99.3 \%)$ & $95(100.0 \%)$ & 0.749 \\
\hline Antihypertensive & $251(83.7 \%)$ & $97(85.1 \%)$ & $120(85.1 \%)$ & $74(77.9 \%)$ & 0.453 \\
\hline Lipid-lowering medications & $298(99.3 \%)$ & $112(98.2 \%)$ & $137(97.2 \%)$ & $95(100.0 \%)$ & 0.140 \\
\hline \multicolumn{6}{|l|}{ Procedural data } \\
\hline \multicolumn{6}{|l|}{ Vessel treated: } \\
\hline LM & $3(1.0 \%)$ & $1(0.9 \%)$ & $4(2.8 \%)$ & $2(2.1 \%)$ & 0.397 \\
\hline LAD & $203(67.7 \%)$ & $77(67.5 \%)$ & $101(71.6 \%)$ & $68(71.6 \%)$ & 0.774 \\
\hline LCX & $97(32.3 \%)$ & $36(31.6 \%)$ & $45(31.9 \%)$ & $32(33.7 \%)$ & 0.989 \\
\hline RCA & $112(37.3 \%)$ & $49(43.0 \%)$ & $53(37.6 \%)$ & $43(45.3 \%)$ & 0.440 \\
\hline Three-vessel or left main & $126(42.0 \%)$ & $50(43.9 \%)$ & $72(51.1 \%)$ & $48(50.5 \%)$ & 0.228 \\
\hline Number of implanted stents & $2(1-2)$ & $2(1-2)$ & $2(1-3)$ & $2(1-3)$ & 0.046 \\
\hline Total stent length $[\mathrm{mm}]$ & $\begin{array}{c}36.00 \\
(24.00-56.00)\end{array}$ & $\begin{array}{c}42.00 \\
(24.00-60.25)\end{array}$ & $\begin{array}{c}48.00 \\
(28.50-64.00)\end{array}$ & $\begin{array}{c}44.00 \\
(29.00-64.00)\end{array}$ & 0.023 \\
\hline Total stent diameter [mm] & $\begin{array}{c}5.25 \\
(3.00-7.00)\end{array}$ & $\begin{array}{c}5.50 \\
(3.00-7.00)\end{array}$ & $\begin{array}{c}5.50 \\
(3.50-7.75)\end{array}$ & $\begin{array}{c}5.50 \\
(3.00-7.75)\end{array}$ & 0.281 \\
\hline
\end{tabular}

Data are median (interquartile range) or number (\%). BMI — body mass index; cTnT — cardiac troponin T; DD - D-dimer; eGFR - estimated glomerular filtration rate; HDL-C — high-density lipoprotein cholesterol; LAD — left anterior descending coronary artery; LCX — left circumflex coronary artery; LDL-C - low-density lipoprotein cholesterol; LM - left main coronary artery; NLR - neutrophil to lymphocyte ratio;

$\mathrm{PCl}$ - percutaneous coronary intervention; RCA — right coronary artery

Table 3 Clinical outcomes of the study population.

\begin{tabular}{lcccccc}
\hline $\begin{array}{l}\text { Outcome (follow-up } \\
\mathbf{4 0} \text { months) }\end{array}$ & $\begin{array}{c}\text { Overall } \\
(\mathbf{n}=650)\end{array}$ & $\begin{array}{c}\text { DD-/NLR- } \\
(\mathbf{n}=300)\end{array}$ & $\begin{array}{c}\text { DD-/NLR+ } \\
(\mathbf{n}=114)\end{array}$ & $\begin{array}{c}\text { DD+/NLR- } \\
(\mathbf{n}=141)\end{array}$ & $\begin{array}{c}\text { DD+/NLR+ } \\
(\mathbf{n}=95)\end{array}$ & $\begin{array}{c}\text { P } \\
\text { MACEs }\end{array}$ \\
\hline $8(15.1 \%)$ & $16(5.3 \%)$ & $19(16.7 \%)$ & $24(17.0 \%)$ & $39(41.1 \%)$ & $<0.001$ \\
All-cause mortality & $28(4.3 \%)$ & $1(0.3 \%)$ & $3(2.6 \%)$ & $9(6.4 \%)$ & $15(15.8 \%)$ & $<0.001$ \\
Cardiac death & $19(2.9 \%)$ & $0(0 \%)$ & $3(2.6 \%)$ & $7(5.0 \%)$ & $9(9.5 \%)$ & $<0.001$ \\
MI & $10(1.5 \%)$ & $0(0 \%)$ & $3(2.6 \%)$ & $2(1.4 \%)$ & $5(5.3 \%)$ & 0.001 \\
TVR & $56(8.6 \%)$ & $14(4.7 \%)$ & $14(12.3 \%)$ & $13(9.2 \%)$ & $15(15.8 \%)$ & 0.003 \\
Stroke & $12(1.8 \%)$ & $1(0.3 \%)$ & $2(1.8 \%)$ & $2(1.4 \%)$ & $7(7.4 \%)$ & 0.001 \\
\hline
\end{tabular}

DD - D-dimer; MACEs - major adverse cardiovascular events; MI - myocardial infarction; NLR - neutrophil to lymphocyte ratio; TVR — target vessel revascularization

EF, eGFR, an increased proportion of three-vessel or left main disease and longer total stent length. There were no significant differences among four groups in terms of BMI, hypertension, dyslipidemia, current smoking, drinking, previous PCI, total cholesterol, triglycerides, LDL-C, HDL-C, and discharge medication.

\section{Clinical outcomes}

During a median follow-up duration of 40 months (IQR: 37-43), the MACEs occurred in $98(15.1 \%)$ patients: 28 events of all-cause death, 12 events of stroke, 10 MIs, and 56 TVRs. Specifi- cally, MACEs occurred in 47 (25.0\%) out of 188 STEMI patients, 10 (19.2\%) out of 52 NSTEMI patients, and $41(10 \%)$ out of 410 unstable angina patients. The MACEs rates were similar between the first two, and both were higher than the last one. The MACEs rates among four groups are presented in Table 3. Patients in DD-/NLR-group had the lowest incidence of MACEs (5.3\%), and those in DD + NLR + group had the highest (41.1\%). Moreover, patients with both high DD and NLR levels had significantly higher rates of all-cause mortality, cardiac death, MI, TVR and stroke than the other three groups. 
Association between baseline

DD, NLR levels and MACEs

To demonstrate the association of baseline DD and NLR levels with MACEs risk, Kaplan-Meier survival curves and Cox regression analyses were performed. Univariate Cox regression analyses showed that age (per 10-year increase), BMI, leucocyte, neutrophil, lymphocyte, NLR, DD (per $0.1 \mu \mathrm{g} / \mathrm{mL}$ increase), HDL-C, eGFR (per $10 \mathrm{~mL} /$ $/ \mathrm{min} / 1.73 \mathrm{~m}^{2}$ increase), $\log$ peak cardiac troponin $\mathrm{T}$, EF (per 10\% increase) and three-vessel or left main disease were predictors for the endpoint of MACEs $(\mathrm{p}<0.15)$. Then, further multivariate Cox analysis revealed that age (per 10-year increase), NLR, DD (per $0.1 \mu \mathrm{g} / \mathrm{mL}$ increase), HDL-C and $\mathrm{EF}$ (per $10 \%$ increase) were independently and significantly associated with MACEs. The same results were obtained using the logistic regression (Suppl. Tables 1, 2).

Four different survival models were then built according to the above-mentioned significant factors. Model 1 was developed based on age (per 10 -year increase), HDL-C, EF (per $10 \%$ increase). Model 2A and 2B added DD and NLR on the basis of model 1, respectively. Model 3 added the combination of DD and NLR (with DD-/NLR- as reference) on the basis of model 1 . After adjusting for variables in model $2 \mathrm{~A}$ and $2 \mathrm{~B}, 1.4$ times and 1.7 times higher risk of MACEs were still observed in elevated levels of DD (adjusted hazard ratio [aHR]: 2.39, 95\% CI: 1.52-3.76, p < 0.001) and elevated levels of NLR (aHR: 2.71, 95\% CI: 1.78-4.11, $\mathrm{p}<0.001$ ) (Suppl. Fig. 1A, B; Table 4). Furthermore, in model 3 , patients in the DD $+/ \mathrm{NLR}+$ group were found to have a significantly higher risk in MACEs than with patients in the DD-/NLRgroup as reference (aHR: 6.19, 95\% CI: 3.30-11.61, $\mathrm{p}<0.001$ ) (Fig. 2, Table 4).

\section{Comparison of predictive efficacy of DD and NLR for MACEs}

Receiver operating characteristic curves were used to evaluate the predictive efficacy of DD, NLR and the combination of DD and NLR (Fig. 3). The AUC of DD to predict MACEs was 0.68 (95\% CI: $0.62-0.73, \mathrm{p}<0.001)$ together with a sensitivity of $64.3 \%$ and a specificity of $68.7 \%$. NLR had an AUC of 0.66 (95\% CI: $0.59-0.72, \mathrm{p}<0.001)$ together with a sensitivity of $59.2 \%$ and a specificity of $72.6 \%$. The combination of DD and NLR achieved the largest AUC (AUC: 0.70, 95\% CI: 0.64-0.76, $\mathrm{p}<0.001)$ in predicting long-term MACEs than DD and NLR alone. The Delong test for comparison of ROC curves revealed that DD had a comparable

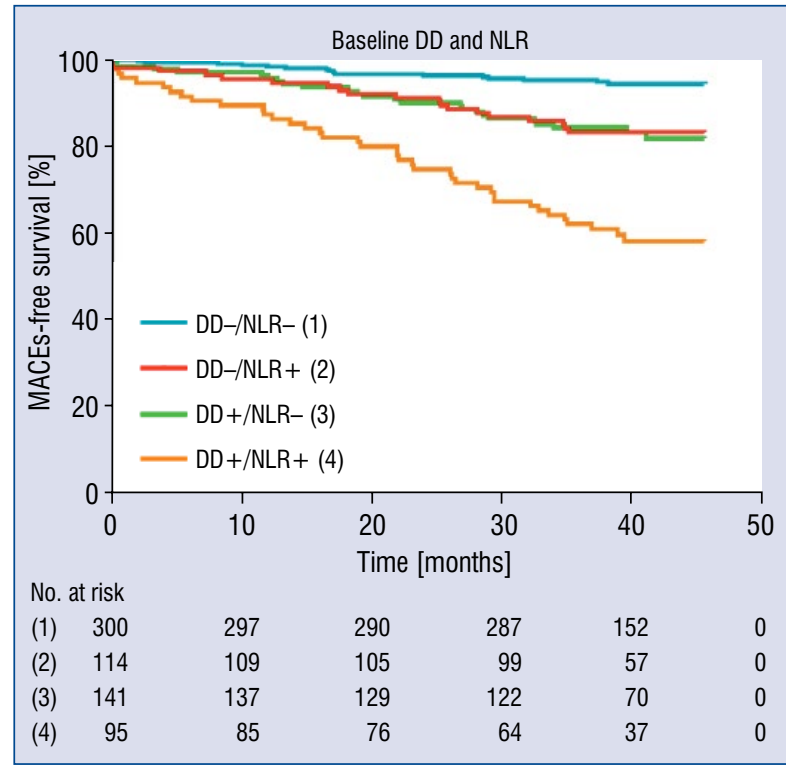

Figure 2. The Kaplan-Meier survival curves according to the combination of D-dimer (DD) and neutrophil to lymphocyte ratio (NLR) in patients with acute coronary syndrome undergoing percutaneous coronary intervention. The median follow-up period was 40 months. For Figure 2, $p<0.001$; MACEs - major adverse cardiovascular events.

value with NLR $(p=0.704)$. Compared with NLR alone, adding DD to NLR resulted in a statistically significant improvement in risk discrimination (AUC) and a nearly significant improvement in reclassification (NRI, IDI) $(\mathrm{p}=0.008$; NRI 4.9\%, $\mathrm{p}=0.11$; IDI $1.6 \%, \mathrm{p}=0.08)$. There was no significant difference in AUC between adding NLR to DD and DD alone, but reclassification shows significant improvement $(\mathrm{p}=0.560$; NRI $17.0 \%$, $\mathrm{p}<0.001$; IDI 5.0\%, $\mathrm{p}<0.001)$.

\section{Comparison of survival models}

The inclusion of DD and NLR in model $2 \mathrm{~A}$ and $2 \mathrm{~B}$ increased the $\mathrm{C}$-index from 0.678 to 0.724 and 0.726 , respectively (likelihood-ratio test $\mathrm{p}<0.001$ for both), indicating a significant improvement compared to model 1 . The $\mathrm{C}$-index for model 3 was 0.750 , revealing a better fit than model $1,2 \mathrm{~A}$ and $2 \mathrm{~B}$ (all $\mathrm{p}<0.001)$.

\section{Discussion}

Considering the large number of ACS patients worldwide and the threat to their lives, how to choose low-cost, easy-to-operate, and repeatable clinical indicators to predict the prognosis of 
Table 4. Different models of major adverse cardiovascular events by multivariate Cox regression analyses.

\begin{tabular}{|c|c|c|c|c|c|c|}
\hline \multirow[t]{2}{*}{ Variable } & \multicolumn{3}{|c|}{ Crude } & \multicolumn{3}{|c|}{ Adjusted* } \\
\hline & HR & $95 \% \mathrm{Cl}$ & $\mathbf{P}$ & HR & $95 \% \mathrm{Cl}$ & $\mathbf{P}$ \\
\hline \multicolumn{7}{|l|}{ Model 1} \\
\hline Age (per 10-year increase) & 1.52 & $1.25-1.85$ & $<0.001$ & 1.33 & $1.05-1.68$ & 0.017 \\
\hline HDL-C & 0.53 & $0.23-1.24$ & 0.145 & 0.34 & $0.14-0.85$ & 0.021 \\
\hline EF (per $10 \%$ increase) & 0.51 & $0.42-0.63$ & $<0.001$ & 0.64 & $0.50-0.82$ & $<0.001$ \\
\hline \multicolumn{7}{|l|}{ Model 2A } \\
\hline Age (per 10-year increase) & 1.52 & $1.25-1.85$ & $<0.001$ & 1.26 & $1.03-1.53$ & 0.022 \\
\hline HDL-C & 0.53 & $0.23-1.24$ & 0.145 & 0.48 & $0.21-1.11$ & 0.086 \\
\hline EF (per $10 \%$ increase) & 0.51 & $0.42-0.63$ & $<0.001$ & 0.59 & $0.48-0.74$ & $<0.001$ \\
\hline DD- & Ref & - & - & Ref & - & - \\
\hline $\mathrm{DD}+$ & 3.47 & $2.30-5.25$ & $<0.001$ & 2.39 & $1.52-3.76$ & $<0.001$ \\
\hline \multicolumn{7}{|l|}{ Model 2B } \\
\hline Age (per 10-year increase) & 1.52 & $1.25-1.85$ & $<0.001$ & 1.39 & $1.16-1.68$ & $<0.001$ \\
\hline HDL-C & 0.53 & $0.23-1.24$ & 0.145 & 0.39 & $0.16-0.92$ & 0.031 \\
\hline EF (per $10 \%$ increase) & 0.51 & $0.42-0.63$ & $<0.001$ & 0.61 & $0.49-0.76$ & $<0.001$ \\
\hline NLR- & Ref & - & - & Ref & - & - \\
\hline NLR+ & 3.39 & $2.26-5.07$ & $<0.001$ & 2.71 & $1.78-4.11$ & $<0.001$ \\
\hline \multicolumn{7}{|l|}{ Model 3} \\
\hline Age (per 10-year increase) & 1.52 & $1.25-1.85$ & $<0.001$ & 1.23 & $1.02-1.49$ & 0.029 \\
\hline HDL-C & 0.53 & $0.23-1.24$ & 0.145 & 0.42 & $0.18-0.97$ & 0.041 \\
\hline EF (per $10 \%$ increase) & 0.51 & $0.42-0.63$ & $<0.001$ & 0.66 & $0.53-0.82$ & $<0.001$ \\
\hline DD-/NLR- & Ref & - & - & Ref & - & - \\
\hline DD-/NLR+ & 3.31 & $1.70-6.43$ & $<0.001$ & 2.76 & $1.40-5.40$ & 0.003 \\
\hline $\mathrm{DD}+/ \mathrm{NLR}-$ & 3.37 & $1.79-6.34$ & $<0.001$ & 2.39 & $1.24-4.63$ & 0.010 \\
\hline $\mathrm{DD}+/ \mathrm{NLR}+$ & 9.40 & $5.25-16.84$ & $<0.001$ & 6.19 & $3.30-11.61$ & $<0.001$ \\
\hline
\end{tabular}

*Adjusted variables include age, body mass index, NLR, DD, HDL-C, estimated glomerular filtration rate, log peak cardiac troponin T, $\mathrm{EF}$, three-vessel or left main disease; $\mathrm{Cl}$ - confidence interval; $\mathrm{DD}$ - D-dimer; $\mathrm{EF}$ - ejection fraction; HDL-C — high-density lipoprotein cholesterol; HR — hazard ratio; NLR - neutrophil to lymphocyte ratio

patients has been a long-standing problem. In the present study, we explored predictive factors and developed models based on DD and NLR to predict MACEs for ACS patients undergoing PCI.

Here, five factors were identified including age, DD, NLR, HDL-C and EF, were associated with MACEs in ACS patients.

Hypercoagulability is the driving factor of atherosclerosis [16], and thrombosis is a further important mechanism for the initiation and development of atherosclerosis and ACS [17]. DD, a degradation product of fibrin molecules, reflects the degree of hypercoagulation and thrombotic activity in the body simultaneously. The LIPID study, which included 7863 patients and up to 16 years of follow-up showed that high baseline DD indicated poor prognoses for all-cause mortality and cardiovascular mortality in patients with stable CHD [2]. Akgul et al. [18] found that high
DD at admission was an independent predictor of in-hospital and 6-month all-cause mortality in STEMI patients receiving primary PCI. AlKhalfan et al. [3] demonstrated that elevated baseline DD was associated with an increased risk of cardiac death, MI, and stroke in patients with ACS, but the median follow-up time of their study was significantly shorter than the present study (6 months vs. 40 months). In another prospective observational study, patients with suspected ACS were followed up for 7 years, finding that high levels of DD were associated with all-cause mortality. However, nearly half of the patients were finally determined to be non-ACS, and only $20 \%$ of the patients received PCI or coronary artery bypass grafting treatment [19]. Thus, according to available research, this is the first study to show that DD levels can predict long-term MACEs for ACS patients undergoing PCI. 


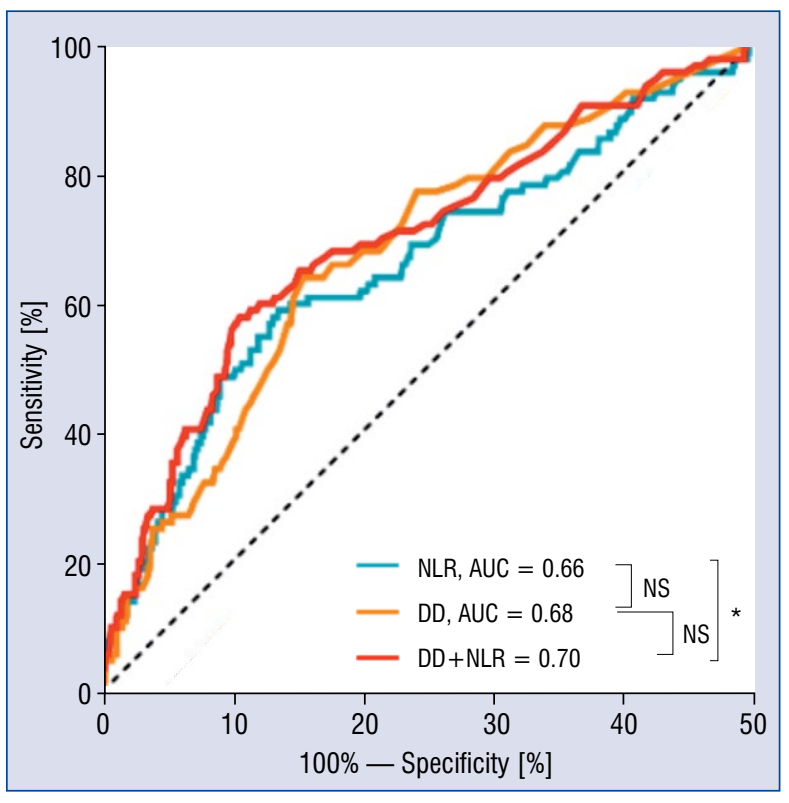

Figure 3. Receiver operating characteristic curves for D-dimer (DD) and neutrophil to lymphocyte ratio (NLR) alone and DD in combination with NLR for predicting longterm major adverse cardiovascular events; AUC — area under curve; ${ }^{*} \mathrm{p}<0.05$; NS - no significant difference.

The mechanism by which elevated DD predicts adverse cardiovascular events is not yet fully understood. It has been documented that elevated DD levels reflect a more coagulable state, accelerate the progression of atherosclerotic lesions, and lead to unstable atherosclerotic plaque activity [20]. In addition, in the LIPID study and Speedwell study, it was found that DD is correlated to C-reactive protein, indicating that the level of DD may also reflect the state of inflammation $[2,19]$. The mechanism may be that DD can mediate and regulate the release of interleukin (IL)-6 from monocytes [21]. IL-6 is an inflammatory factor and a clinical study has shown that inhibiting the IL-6 signaling pathway via a specific drug was associated with a reduced risk of cardiovascular events and all-cause mortality in patients [22]. Therefore, we reasoned that baseline DD levels could reflect both thrombotic activity and inflammation, making it a good predictor. In the current study, baseline DD was an independent predictor of long-term MACEs in patients with ACS after PCI and had a comparable efficiency for differentiating MACEs to NLR. However, owing to the missing data on inflammation-related indicators, association between DD and inflammation could not be verified. Further research can be anticipated to expound on the relationship between the two.
Neutrophil to lymphocyte ratio was calculated by dividing the neutrophil count by the lymphocyte count, which represents the dynamic balance of neutrophil and lymphocyte and reflects the inflammation state of the body. Previous observational epidemiologic studies have described the relationship between an elevated neutrophil count and increased cardiovascular risk in patients with stable angina and ACS [23, 24]. Neutrophil are involved in the progression of many aspects of cardiovascular pathophysiology, including atherosclerosis and thrombosis, eventually leading to ACS. There are three main mechanisms for how neutrophil mediates atherosclerosis: (1) neutrophils can accumulate in atherosclerotic lesions and enhance plaque inflammation through degranulation of various proteins and enzymes, (2) neutrophils can recruit themselves, oxidize LDL-C, exacerbate endothelial cell dysfunction and cause plaque vulnerability by releasing reactive oxygen species, and (3) neutrophils contribute to plaque rupture by eroding the fibrous cap via proteinases and elastase. It was also reported that neutrophils can also activate coagulation by releasing neutrophil extracellular traps [25]. Neutrophil extracellular traps then provide a hypercoagulable microenvironment, induce platelet activation, bind clotting factors and promote thrombosis [26].

Apart from neutrophils, lymphocytes also play an important role in the inflammatory reaction during the atherosclerotic process. Low lymphocyte count has been demonstrated to be associated with increased risk of adverse events in various cardiovascular disease such as stable CHD, ACS and heart failure [23, 27, 28]. Experimental study revealed that increased lymphocytes apoptosis may be responsible for the association between low lymphocyte count and poor prognosis of cardiovascular diseases [29]. Apoptosis of lymphocytes that occurs in atherosclerotic lesions promotes atherosclerotic plaque growth, leads to lipid core development, and ultimately results in thrombotic events. Spontaneous lymphocyte apoptosis was found both in criminal plaque and peripheral blood, and it was more severe in ACS patients [30]. Therefore, by integrating neutrophil and lymphocyte counts simultaneously, NLR as an indicator of inflammation was found to have a more significant correlation in predicting long-term adverse cardiac outcomes in patients with ACS. In the present study, the incidence of MACEs was significantly higher in patients with elevated NLR, which is consistent with findings of previous studies [7, 8, 31]. It is also worth mentioning that not only the non-infection- 
-associated hyperinflammation status in the body is related to MACEs, but inflammation caused by infectious diseases, such as influenza, has also been confirmed by clinical study to be related to acute myocardial infarction [32].

Additionally, it was found that older age, reduced $\mathrm{EF}$ and low HDL-C level were also associated with increased risks in patients with ACS undergoing PCI. Advanced age and decreased EF are both known risk factors for increased mortality in patients with ACS. When it comes to HDL-C, a low level of HDL-C had been shown to be a good prognostic marker for predicting adverse cardiac events in patients with ACS [33]. In addition to the classic reverse cholesterol transport effect of HDL-C, recent studies have shown that HDL-C can also exert antithrombotic effects and downregulate thrombin generation [34], as well as anti-inflammatory and regulating neutrophil function $[35,36]$. Consistently, the current study showed that high HDL-C level was a protective factor in patients with ACS after PCI, and the combined value of DD and NLR for predicting MACEs was independent of HDL-C level.

Based on these predictive factors, survival models were further developed to predict MACEs in patients with ACS receiving PCI, which can quantify predictive factors for patients, help clinicians to assess prognosis as soon as possible and design appropriate strategies for each patient.

\section{Limitations of the study}

There are some limitations that need to be considered in the current study. First, the sample size was relatively small and was based on a single center, which might bring about selection bias. Second, in this retrospective study, 53 (7.5\%) patients' follow-up data were not available and the data collection might have missed some other important biomarkers such as C-reactive protein and B-type natriuretic peptide, so we were not able to investigate the relation between these markers and clinical outcomes, which may result in potential underpowering of some analyses. Third, we only compared the DD levels and NLR at admission, because data on the dynamic changes over time was limited. Considering the above-reported limitations, further prospective, well-designed and multicentric studies in larger scale are warranted to confirm the present findings.

\section{Conclusions}

Admission DD and NLR are associated with long-term MACEs in ACS patients receiving PCI.
The combination of the two may provide a more sensitive factor and help further optimize risk stratification and treatments in patients with ACS after PCI.

\section{Acknowledgments}

This work was supported by grants from the National Natural Science Foundation of China (No. 81770361), and Jiangsu Province's Key Provincial Talents Program (No. ZDRCA201604).

\section{Conflict of interest: None declared}

\section{References}

1. Roth GA, Johnson C, Abajobir A, et al. Global, regional, and national burden of cardiovascular diseases for 10 causes, 1990 to 2015. J Am Coll Cardiol. 2017; 70(1): 1-25, doi: 10.1016/j. jacc.2017.04.052, indexed in Pubmed: 28527533.

2. Simes J, Robledo KP, White HD, et al. LIPID Study Investigators. D-Dimer predicts long-term cause-specific mortality, cardiovascular events, and cancer in patients with stable coronary heart disease: LIPID study. Circulation. 2018; 138(7): 712-723, doi: 10.1161/CIRCULATIONAHA.117.029901, indexed in Pubmed: 29367425.

3. AlKhalfan F, Kerneis M, Nafee T, et al. D-Dimer levels and effect of rivaroxaban on those levels and outcomes in patients with acute coronary syndrome (an ATLAS ACS-TIMI 46 trial substudy). Am J Cardiol. 2018; 122(9): 1459-1464, doi: 10.1016/j. amjcard.2018.07.032, indexed in Pubmed: 30217378.

4. Ross R. Atherosclerosis--an inflammatory disease. N Engl J Med. 1999; 340(2): 115-126, doi: 10.1056/NEJM199901143400207, indexed in Pubmed: 9887164.

5. Falk E, Nakano M, Bentzon JF, et al. Update on acute coronary syndromes: the pathologists' view. Eur Heart J. 2013; 34(10): 719-728, doi: 10.1093/eurheartj/ehs411, indexed in Pubmed: 23242196.

6. Wada H, Dohi T, Miyauchi K, et al. Pre-procedural neutrophil-tolymphocyte ratio and long-term cardiac outcomes after percutaneous coronary intervention for stable coronary artery disease. Atherosclerosis. 2017; 265: 35-40, doi: 10.1016/j.atherosclerosis.2017.08.007, indexed in Pubmed: 28843126.

7. Gul M, Uyarel H, Ergelen M, et al. Predictive value of neutrophil to lymphocyte ratio in clinical outcomes of non-ST elevation myocardial infarction and unstable angina pectoris: a 3-year follow-up. Clin Appl Thromb Hemost. 2014; 20(4): 378-384, doi: 10.1177/1076029612465669, indexed in Pubmed: 23144177.

8. Han YC, Yang TH, Kim DI, et al. Neutrophil to lymphocyte ratio predicts long-term clinical outcomes in patients with st-segment elevation myocardial infarction undergoing primary percutaneous coronary intervention. Korean Circ J. 2013; 43(2): 93-99, doi: 10.4070/kcj.2013.43.2.93, indexed in Pubmed: 23508782.

9. Benites-Zapata VA, Hernandez AV, Nagarajan V, et al. Usefulness of neutrophil-to-lymphocyte ratio in risk stratification of patients with advanced heart failure. Am J Cardiol. 2015; 115(1): 57-61, doi: 10.1016/j.amjcard.2014.10.008, indexed in Pubmed: 25456873.

10. Tamhane UU, Aneja S, Montgomery D, et al. Association between admission neutrophil to lymphocyte ratio and outcomes 
in patients with acute coronary syndrome. Am J Cardiol. 2008; 102(6): 653-657, doi: 10.1016/j.amjcard.2008.05.006, indexed in Pubmed: 18773982.

11. Thygesen K, Alpert JS, Jaffe AS, et al. Fourth Universal Definition of Myocardial Infarction (2018). J Am Coll Cardiol. 2018; 72(18): 2231-2264, doi: 10.1016/j.jacc.2018.08.1038, indexed in Pubmed: 30153967.

12. Ibanez B, James S, Agewall S, et al. ESC Scientific Document Group. 2017 ESC Guidelines for the management of acute myocardial infarction in patients presenting with ST-segment elevation: The Task Force for the management of acute myocardial infarction in patients presenting with ST-segment elevation of the European Society of Cardiology (ESC). Eur Heart J. 2018; 39(2): 119-177, doi: 10.1093/eurheartj/ehx393, indexed in Pubmed: 28886621.

13. Amsterdam EA, Wenger NK, Brindis RG, et al. 2014 AHA/ /ACC Guideline for the Management of Patients With Non-ST-Elevation Acute Coronary Syndromes: A Report of the American College of Cardiology/American Heart Association Task Force on Practice Guidelines. J Am Coll Cardiol. 2014; 64: e139-e228, doi: 25260718, indexed in Pubmed: 10.1016/j.jacc.2014.09.017.

14. Hankey G. Stroke. Lancet. 2017; 389(10069): 641-654, doi: 10.1016/s0140-6736(16)30962-x.

15. Cutlip DE, Windecker S, Mehran R, et al. Academic Research Consortium. Clinical end points in coronary stent trials: a case for standardized definitions. Circulation. 2007; 115(17): 2344-2351, doi: 10.1161/CIRCULATIONAHA.106.685313, indexed in Pubmed: 17470709.

16. Borissoff JI, Spronk HMH, ten Cate H. The hemostatic system as a modulator of atherosclerosis. N Engl J Med. 2011; 364(18): 1746-1760, doi: 10.1056/NEJMra1011670, indexed in Pubmed: 21542745.

17. Fuster V, Badimon L, Badimon J, et al. The pathogenesis of coronary artery disease and the acute coronary syndromes. N Engl J Med. 1992; 326(5): 310-318, doi: 10.1056/ nejm199201303260506.

18. Akgul O, Uyarel H, Pusuroglu H, et al. Predictive value of elevated $\mathrm{D}$-dimer in patients undergoing primary angioplasty for ST elevation myocardial infarction. Blood Coagul Fibrinolysis. 2013; 24(7): 704-710, doi: 10.1097/MBC.0b013e3283610396, indexed in Pubmed: 23571687.

19. Lowe GD, Yarnell JW, Rumley A, et al. C-reactive protein, fibrin D-dimer, and incident ischemic heart disease in the Speedwell study: are inflammation and fibrin turnover linked in pathogenesis? Arterioscler Thromb Vasc Biol. 2001; 21(4): 603-610, doi: 10.1161/01.atv.21.4.603, indexed in Pubmed: 11304479.

20. Figueras J, Monasterio Y, Lidón RM, et al. Thrombin formation and fibrinolytic activity in patients with acute myocardial infarction or unstable angina: in-hospital course and relationship with recurrent angina at rest. J Am Coll Cardiol. 2000; 36(7): 2036-2043, doi: 10.1016/s0735-1097(00)01023-8, indexed in Pubmed: 11127437.

21. Lowe GDO, Rumley A, McMahon AD, et al. West of Scotland Coronary Prevention Study Group. Interleukin-6, fibrin D-dimer, and coagulation factors VII and XIIa in prediction of coronary heart disease. Arterioscler Thromb Vasc Biol. 2004; 24(8): 1529-1534, doi: 10.1161/01.ATV.0000135995.39488.6c, indexed in Pubmed: 15205218.

22. Ridker PM, Libby P, MacFadyen JG, et al. Modulation of the interleukin-6 signalling pathway and incidence rates of atherosclerotic events and all-cause mortality: analyses from the
Canakinumab Anti-Inflammatory Thrombosis Outcomes Study (CANTOS). Eur Heart J. 2018; 39(38): 3499-3507, doi: 10.1093/ eurheartj/ehy310, indexed in Pubmed: 30165610.

23. Horne BD, Anderson JL, John JM, et al. Intermountain Heart Collaborative Study Group. Which white blood cell subtypes predict increased cardiovascular risk? J Am Coll Cardiol. 2005; 45(10): 1638-1643, doi: 10.1016/j.jacc.2005.02.054, indexed in Pubmed: 15893180.

24. Dragu R, Huri S, Zukermann R, et al. Predictive value of white blood cell subtypes for long-term outcome following myocardial infarction. Atherosclerosis. 2008; 196(1): 405-412, doi: 10.1016/j. atherosclerosis.2006.11.022, indexed in Pubmed: 17173924.

25. Hoyer FF, Nahrendorf M. Neutrophil contributions to ischaemic heart disease. Eur Heart J. 2017; 38(7): 465-472, doi: 10.1093/ eurheartj/ehx017, indexed in Pubmed: 28363210.

26. Gaul DS, Stein S, Matter CM. Neutrophils in cardiovascular disease. Eur Heart J. 2017; 38(22): 1702-1704, doi: 10.1093/ eurheartj/ehx244, indexed in Pubmed: 30052884.

27. Ommen SR, Gibbons RJ, Hodge DO, et al. Usefulness of the lymphocyte concentration as a prognostic marker in coronary artery disease. Am J Cardiol. 1997; 79(6): 812-814, doi: 10.1016/ s0002-9149(96)00878-8, indexed in Pubmed: 9070569.

28. Acanfora D, Gheorghiade M, Trojano L, et al. Relative lymphocyte count: a prognostic indicator of mortality in elderly patients with congestive heart failure. Am Heart J. 2001; 142(1): 167-173, doi: 10.1067/mhj.2001.115792, indexed in Pubmed: 11431674.

29. Stoneman VEA, Bennett MR. Role of apoptosis in atherosclerosis and its therapeutic implications. Clin Sci (Lond). 2004; 107(4): 343-354, doi: 10.1042/CS20040086, indexed in Pubmed: 15230690.

30. Pasqui AL, Di Renzo M, Bova G, et al. T cell activation and enhanced apoptosis in non-ST elevation myocardial infarction. Clin Exp Med. 2003; 3(1): 37-44, doi: 10.1007/s102380300014, indexed in Pubmed: 12748878.

31. Park JJ, Jang HJ, Oh IY, et al. Prognostic value of neutrophil to lymphocyte ratio in patients presenting with ST-elevation myocardial infarction undergoing primary percutaneous coronary intervention. Am J Cardiol. 2013; 111(5): 636-642, doi: 10.1016/j. amjcard.2012.11.012, indexed in Pubmed: 23273716.

32. Kwong JC, Schwartz KL, Campitelli MA, et al. Acute myocardial infarction after laboratory-confirmed influenza infection. N Engl J Med. 2018; 378(4): 345-353, doi: 10.1056/NEJMoa1702090, indexed in Pubmed: 29365305.

33. Wolfram RM, Brewer HB, Xue Z, et al. Impact of low high-density lipoproteins on in-hospital events and one-year clinical outcomes in patients with non-ST-elevation myocardial infarction acute coronary syndrome treated with drug-eluting stent implantation. Am J Cardiol. 2006; 98(6): 711-717, doi: 10.1016/j. amjcard.2006.04.006, indexed in Pubmed: 16950168.

34. Griffin JH, Kojima K, Banka CL, et al. High-density lipoprotein enhancement of anticoagulant activities of plasma protein $\mathrm{S}$ and activated protein C. J Clin Invest. 1999; 103(2): 219-227, doi: 10.1172/JCI5006, indexed in Pubmed: 9916134.

35. Besler C, Heinrich K, Rohrer L, et al. Mechanisms underlying adverse effects of HDL on eNOS-activating pathways in patients with coronary artery disease. J Clin Invest. 2011; 121(7): 2693-2708, doi: 10.1172/JCI42946, indexed in Pubmed: 21701070.

36. Baetta R, Corsini A. Role of polymorphonuclear neutrophils in atherosclerosis: current state and future perspectives. Atherosclerosis. 2010; 210(1): 1-13, doi: 10.1016/j.atherosclerosis.2009.10.028, indexed in Pubmed: 19931081. 